\title{
La convergencia entre América Latina y China
}

\author{
Jorge Castro*
}

\section{INTRODUCCIÓN}

América Latina crece este año $6 \%$ o más, encabezada por Brasil, que se expande $8.5 \%$, tras haber aumentado $8.9 \%$ en los primeros seis meses de 2010. Se supera así el récord establecido durante el boom 2003 / 2008, en que creció 5.2\%, con inflación menor a un dígito. Lo notable del auge de 2010 es que todos los países de América del Sur tienen la misma estructura de comercio exterior, con más de la mitad de sus exportaciones constituidas por materias primas (soja, cobre, mineral de hierro): Brasil, 56\%; Chile, Colombia, Perú, $70 \%$ / 80\%.

China se ha convertido también en el mayor socio comercial de Brasil, Chile y Perú y, considerado como país individual, en el principal destino de las exportaciones agroalimentarias argentinas. La correlación entre el precio de las expor-

Presidente del Instituto de Planeamiento Estratégico (IPE, Buenos Aires) taciones de América del Sur y la demanda industrial china es más del $60 \%$.

El efecto más importante de la irrupción de China/Asia en el comercio internacional es la modificación que ha provocado en los términos de intercambio globales (relación entre el precio de las exportaciones y el precio de las importaciones). Por eso, para el resto de los países emergentes, ante todo los de América del Sur, desaparecieron las ventajas comparativas en los productos industriales trabajo-intensivos y reaparecieron en gran escala las ventajas comparativas en la producción de commodities.

El resultado es que la estrategia de desarrollo que le impone a América del Sur la nueva estructura de la acumulación global con eje en China surge ahora, por necesidad, de la transformación irreversible de los términos de intercambio.

China se convierte este año en la principal inversora directa en Brasil, 
como ya lo es en Perú (Camisea / US\$ 4.300 millones), con inversiones en minería, acero, industria automotriz e infraestructura. La República Popular es la mayor exportadora mundial de capitales (11\% del PBI en $2008 /$ US\$ 350.000 millones), con inversiones directas en el exterior que alcanzarían a US\$ 100.000 millones en 2010 (US\$ 56.500 millones en 2009).

China compraría parte de la nueva oferta accionaria de Petrobras por US\$ 32.000 millones, parte de una ampliación de capital de US\$ 82.000 que integra millones, con un plan de inversión de US\$224.000 en 5 años para desarrollar el Pre-Sal.

Lo que está ocurriendo es que la República Popular China comienza a reciclar su exceso de ahorro hacia otros países emergentes, al igual que lo hizo en los últimos 15 años con Estados Unidos, cuando adquirió títulos del Tesoro por US\$ 1.5 mil millones.

La contrapartida es que las exportaciones brasileñas a China aumentaron $60.3 \%$ en los primeros 6 meses del año, a un ritmo mayor que el de la región asiática. La integración creciente entre China y Brasil es parte del incremento del comercio Sur-Sur, una de las manifestaciones centrales del vuelco de la demanda mundial desde los países avanzados a los emergentes. El comercio Sur-Sur, primordialmente Asia/ América del Sur, asciende a $43.1 \%$ del total del comercio mundial en 2010.

El problema de América del Sur no es la estructura de sus exportaciones basada en las materias primas, sino su dé- bil crecimiento potencial de largo plazo, su baja tasa de inversión y su nula o negativa productividad. América del Sur no está castigada por la «maldición de las materias primas»-apreciación de la moneda e incapacidad de exportar de los otros sectores de la economía-, sino por su incapacidad histórica para incrementar la productividad a través de un proceso incesante de aperturas y reformas.

Esto es lo que crea una estructura económica heterogénea y divergente entre el sector de materias primas y el resto. En cambio, cuando la productividad del conjunto es alta, la disponibilidad en gran escala de materias primas es una bendición. Es el caso de Australia, Canadá, los países escandinavos y Estados Unidos, entre otros.

\section{LA ECONOMÍA NORTEAMERICANA POS CRISIS 2008/2009}

La economía norteamericana mantiene una baja tasa de crecimiento, cercana al estancamiento: $2 \%$ anual en el tercer trimestre, $1.7 \%$ en el segundo, que sería no más de $2.7 \%$ en el año. Por eso la tasa de desocupación se mantiene estable en los últimos tres meses: 9.6\%; es lo que sucede cuando el PBI se expande solo entre $2.5 \%$ y $2.8 \%$ anual.

Pero las grandes empresas industriales, todas ellas trasnacionales, encabezadas por las de alta tecnología, tienen niveles record de ganancias en el tercer trimestre, sobre todo por su facturación excepcional en el mundo emergente 
Jorge CASTro • La convergencia entre América Latina

(China, India, Brasil), con ventas que aumentaron entre $10 \% /$ y $15 \%$ respecto del trimestre anterior (en Estados Unidos la facturación creció 4\%).

Las empresas trasnacionales (ETN) norteamericanas producen y venden fuera de Estados Unidos entre 4 y 6 veces más de lo que exportan desde el territorio estadounidense. Es el caso de todas ellas, desde Alcoa, John Deere, Caterpillar y Boeing, hasta Apple, Microsoft y muchas más. Las ETN son $42 \%$ del PBI industrial y casi $80 \%$ de la alta tecnología.

La productividad en Estados Unidos ha sido extraordinariamente elevada entre 2008 y 2010, en los dos años de crisis y recesión y en la débil recuperación posterior: en los últimos 12 meses creció más de $6 \%$ y en el tercer trimestre aumentó $2.5 \%$ anual, sumado al incremento de $3.7 \%$ del período previo. Por eso el producto per cápita no cayó tanto como en los otros países avanzados. En Estados Unidos disminuyó $(-5.4 \%)$ entre septiembre 2008 y julio 2009; en la Zona Euro (-8\% / -9\%) en Japón $-15 \%$. Pero lo que aumentó extraordinariamente fue la desocupación, que se duplicó entre 2008 y 2010 (de $4.6 \%$ a $9.6 \%$ ). Es el resultado de la recesión 2008-2009, cruzada por la alta productividad.

También es récord el lucro de las empresas estadounidenses: aumentó $70 \%$ en los primeros 6 meses de 2010 .

El ciclo capitalista no se define por el aumento de la demanda o el crecimiento del producto, sino por la tasa de ganancia. La punta de lanza de la rentabilidad estadounidense corresponde a las empresas de alta tecnología. Las tres principales (Apple, Google, Microsoft) tuvieron ingresos netos de US\$ 5.200 millones, US\$ 4.600 millones y US\$ 14.600 millones, respectivamente; y este año los habrían doblado. El nivel de capitalización en el mercado de Microsoft -US\$230.400 millones en 2009- creció este año entre $20 \%$ y $30 \%$ y lo mismo ocurre con las otras dos que siguen a las tres principales: Amazon y Facebook. Por eso tienen una extraordinaria liquidez y una enorme capacidad de compra de empresas periféricas, en Estados Unidos y en el resto del mundo.

Lo notable es que las ETN pierden posiciones en el mercado estadounidenses porque se desprenden de sus tareas accesorias y multiplican sus inversiones directas en el exterior (downsizing, outsourcing ). Su participación en el PBI era $32 \%$ en 1977 y ahora es $21 \%$; y su inversión en el exterior se multiplicó por dos en ese período. Las ETN responden por $67 \%$ de las exportaciones estadounidenses y $40 \%$ de las importaciones; y 3/5 de las exportaciones industriales son partes y componentes, no productos terminados.

Hay una nueva revolución tecnológica en Estados Unidos, en que el acceso a Internet, y por lo tanto las operaciones de las empresas en la red, se realizan a través de una pluralidad de instrumentos individualizados y portables, y no más mediante computadoras personales (cloud computing).

Flujo de capitales hacia los países emergentes 
Los países emergentes reciben este año US\$ 825.000 millones como saldo neto de capitales privados provenientes del mundo avanzado. Es $40 \%$ más que el año pasado (US\$ 581.000 millones). Se prevé que ese flujo de capitales tenga en 2011 un piso de US\$ 833.000 millones. De los que se recibe este año, US\$ 186.000 millones son compras de acciones (equities), de las que Asia emergente recibe US\$93.000 millones; y América Latina, US\$72.000 millones. Brasil, solo, atrae US\$58.000 a través de la Bolsa de San Pablo.

La inversión extranjera directa (IED) de las empresas trasnacionales es un componente fundamental de esta ola de capitales que se vuelca al mundo emergente: asciende este año a US\$ 367.000 millones ( $45 \%$ del flujo global). China obtiene US\$ 90.000 millones de IED; India, US\$ 40.000 millones; y Brasil, US\$50.000.

Los grandes inversores institucionales aumentaron su presencia estratégica en los mercados emergentes, llevando sus activos de $5 \%$ a $20 \%$ o más.

Este no es un fenómeno monetario (usualmente denominado «especulativo») sino real. El flujo principal de capitales del sistema financiero internacional se adelanta -y financia- la expansión del comercio global, que este año crece $13.5 \%$ (OMC); y también se proyecta hacia los mercados internos, al financiar el consumo interno de los países emergentes, en relación directa con la apreciación de sus monedas, que se fortalecen según el flujo de capitales que reciben.
Así se consolida el mecanismo de acumulación global surgido de la crisis -o nueva división internacional del trabajo-, con eje en los países emergentes (China, India, Brasil), que crecen sobre la base de su demanda interna y el consumo interno. Este gigantesco flujo de capitales guía la transición del sistema mundial hacia un nuevo equilibrio histórico, en el que los países emergentes crecerían a través de su demanda doméstica, mientras que los avanzados, previa drástica reestructuración tecnológica y organizativa, lo harían mediante las exportaciones y el auge de la productividad de todos los factores, es decir, de la innovación.

Este flujo de capitales revela que la demanda global pasa de los países deficitarios en sus cuentas externas, como es el caso de Estados Unidos, a los superavitarios en ellas, China en primer lugar.

Esta es la transición central del sistema mundial en la etapa post-crisis 2008/2009 en un sistema capitalista plenamente integrado, como el que reveló la crisis, el saldo neto de las cuentas externas del mundo emergente y el avanzado (China/ Estados Unidos) es igual a cero. Por eso, lo decisivo para destrabar el impasse de los «desequilibrios comerciales» de la economía mundial -eje crítico de la situación actual- es que China crezca sobre la base de su demanda interna y arrastre así, mediante el aumento estructural de sus importaciones, la recuperación del mundo avanzado a través de sus ventas externas. 
En los primeros 10 meses del año las importaciones chinas crecieron $40.5 \%$ anual, mientras que las exportaciones aumentaron $32.7 \%$. El resultado es que el superávit comercial es ahora menos del $4 \%$ del PBI. El mundo avanzado enfrenta un largo período histórico de débil crecimiento económico, alta desocupación y honda reestructuración organizativa y tecnológica.

Los capitales no se caracterizan por su optimismo o pesimismo, sino por su percepción de riesgos y oportunidades. La Conference Board estima que en dos años (2012) China sobrepasa a Estados Unidos en capacidad de compra interna (PPP). Agrega que el PBI chino sería 24\% del mundial en 2020, mientras que Estados Unidos se reduciría a $14.6 \%$. El vuelco de capitales al mundo emergente no tiene nada de «especulativo».

\section{La CUESTión CLAVE DE América LATINA ES LA CONVERGENCIA CON ASIA}

Brasil lidera el crecimiento latinoamericano: el PBI aumentó $8.9 \%$ anual en los primeros seis meses del año, arrastrado por una demanda doméstica que experimentó un alza de $12 \%$ en ese período. El crecimiento de América Latina es el doble de Estados Unidos y cuatro veces el de la Zona Euro.

México es el país de la región que tuvo la mayor contracción en 2009 (-6.5\%), y este año crecería $5 \%$ o más.

A la cabeza de la recuperación mexicana se encuentra la industria au- tomotriz: sus exportaciones a Estados Unidos se incrementaron $56.7 \%$ en abril y alcanzaron a 91.300 millones de dólares en los primeros cuatro meses del año, un alza de $35 \%$ con respecto al año anterior. Por eso, las reservas del Banco Central superaron los 100.000 millones de dólares, récord histórico.

También el porcentaje mexicano en el total de las importaciones estadounidenses alcanzó un nivel inédito de $12.3 \%$ ( $11 \%$ en 2009$)$. No solo aumentan las exportaciones a Estados Unidos, sino que se modifica su naturaleza.

Venezuela es el país de América Latina que tiene este año la mayor contracción. En el primer trimestre experimentó una caída de $5.8 \%$, una de las mayores del mundo. En ese período, la inversión privada se derrumbó $27.9 \%$. Una caída semejante parece indicar que en Venezuela, más que una recesión, hay destrucción sistemática de capacidades productivas.

Este es el segundo año de recesión en Venezuela: en 2009 el PBI cayó $3.3 \%$ y esto ocurrió a pesar de que el precio del petróleo alcanzó los 82 dólares el barril. Las exportaciones no petroleras disminuyeron $50 \%$ el año pasado, prácticamente desaparecieron. Hoy Venezuela es estrictamente un país mono productor.

América Latina ha experimentado dos puntos de inflexión histórica. El primero fue en 1991 cuando la caída por implosión de la Unión Soviética unificó el sistema y el capitalismo, virtualmente global desde su origen, lo fue efectivamente. El resultado fue que el sistema 
se extendió al mundo entero y en los países en desarrollo la incorporación se produjo al mismo tiempo (1991) desde Asia a América del Sur.

Luego transcurrieron dos décadas de crisis y reformas, hasta que en 2007/2008 se alcanzó un mínimo de estabilidad política y fiscal que le permitió a la región emerger exitosamente del segundo punto de inflexión del sistema mundial: la crisis global 2008/2009. El primero fue provocado por un acontecimiento geopolítico: el colapso de la Unión Soviética y la unificación del sistema. El segundo es obra de la irrupción de un nuevo mecanismo de acumulación global, con eje en China y los países asiáticos.

La cuestión para América del Sur en la próxima década es la convergencia con Asia, y su integración plena en el nuevo mecanismo de acumulación global, cuyo eje estratégico es China.

La fractura de fondo en la región -más allá de ideologías, carismas o personalidades- es la que existe entre la opción por volcarse a la nueva tendencia histórica de convergencia con Asia/ China, o negarla y rechazarla por el motivo que sea.

\section{La Situación de la Argentina}

La economía argentina creció $2.5 \%$ anual entre 1950 y 2008 (1.1\% de aumento del PBI per cápita). Pero entre 1970 y 1990 el PBI por habitante se hundió $(-15 \%)$. De pronto, la tendencia cambió y entre 1990 y 2010 el ingreso per cápita creció $60 \%$ y se produjo un auge notable con un aumento decisivo de la relación exportaciones/PBI.

¿Qué tendencia apareció a partir de 1990? Es la producción agroalimentaria encabezada por la soja (harinas y aceites). Lo que sucede en la Argentina es que por primera vez en 70 años dispone de una producción que al mundo le interesa comprar, sobre todo al sector más vigoroso del capitalismo globalizado: China/Asia. Por eso, han aparecido ahora superávits externos capaces de financiar las importaciones necesarias para sostener una alta tasa de crecimiento.

El crecimiento económico argentino es históricamente un proceso de acumulación en condiciones de alta incertidumbre. Esta incertidumbre deriva de la crónica crisis política y de la fragilidad estructural del sector externo (carencia de dólares suficientes).

Este segundo factor de incertidumbre no está hoy presente, sobre todo gracias a los sectores automotriz y agroalimentario. Sigue en pie el primero -la crisis política-, con fuerza acentuada en 2010. Esta es la primera vez desde 1974 en que una crisis política, al no coincidir con un frágil sector externo, no provoca una crisis fiscal, monetaria y económica.

La cuestión que enfrenta este año la Argentina es cómo resolver su crisis política y al mismo tiempo organizar su producción -inversión, incentivos, infraestructura, instituciones- para multiplicar los productos que el mercado mundial le requiere y que son los 
Jorge CASTro • La convergencia entre América Latina

que el país produce y exporta con uno de los mayores niveles de productividad / competitividad del mundo.

\section{REFERENCIAS BIBLIOGRÁFICAS}

Broda, Miguel Ángel, (2010) Ciclo de análisis económico y político, Estudio Broda \& Asociados.

Castro, Jorge, (2000) La gran década, Buenos Aires, Editorial Sudamericana

FAO, The State of Agricultural Commodity Markets 2009.
North, Douglas, (1994) Economic Performance through Time, The American Economic Review, Vol. 84, No. 3.

NorTH, Douglas, John Joseph Wallis y Barry R. Weingast, (2006) A conceptual framework for interpreting recorded human history, Cambridge,

Working PAPER 12795, National Bureau of Economic Research.

OCDE, (2010) Perspectives on Global Development 2010. Shifting Wealth.

OCDE, (2009) Economic Outlook No. 86, Noviembre.

OCDE, (1997) Argentina en el siglo XX: Crónica de un crecimiento anunciado. 